\title{
USEFULNESS OF LEFT VENTRICLE DYSSYNCHRONY ASSESSMENT BEFORE CARDIAC RESYNCHRONIZATION IMPLANTATION
}

\author{
Jolana Lipoldova, Eva Ozabalova, Jaroslav Meluzin, Miroslav Novak, Jiri Vitovec
}

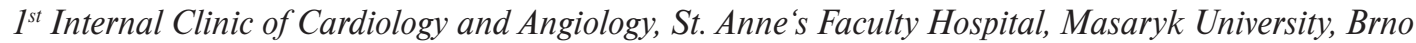 \\ E-mail:jolana.lipoldova@fnusa.cz
}

Received: September 15, 2009; Accepted: February 4, 2010

Key words: Cardiac resynchronization therapy/Left ventricle dyssynchrony/Prediction of CRT response

Background. A number of trials have demonstrated the effect of cardiac resynchronization therapy (CRT) on functional improvement and reversed left ventricle remodeling. Meeting contemporary guidelines approximately 30$40 \%$ of patients do not respond to CRT (non-responders).

Aim. To quantify the predictive ability of basal QRS width and basal echocardiographic parameters of left ventricle contraction dyssynchrony in our group of CRT patients. To compare effectiveness of these parameters assessment in patients with ischemic (iCMP) and non-ischemic cardiomyopathy (niCMP) and with sinus rhythm (SR) and atrial fibrillation (AF).

Patients and methods. 194 patients after successful introduction of CRT device were evaluated. Evaluation of NYHA function class, QRS width and echocardiographic parameters including parameters of left ventricle contraction dyssynchrony (SPWMD: septal-to-posterior wall motion delay, Ts-sep-lat: time interval between maximum of systolic movement of septum and lateral wall using tissue Doppler imaging, IVMD: interventricular mechanical delay) performed before implantation and 3 months after implantation of CRT device.

Results. Responder (improved in NYHA class after CRT) rate was $61 \%$. SR patients showed higher benefit compared to AF patients (responder rate 63\% vs. 52\%, p<0.05). Narrowing of QRS width after CRT was observed only in responders. SPWMD and Ts-sep-lat decreased after CRT in all subgroups. SPWMD dyssynchrony (SPMWD $\geq$ $130 \mathrm{~ms}$ ) reduction after CRT was more expressed in niCMP population. Ts-sep-lat dyssynchrony (Ts-sep-lat $\geq 65 \mathrm{~ms}$ ) reduction after CRT was more expressed in SR patients. IVMD (IVMD $\geq 60 \mathrm{~ms}$ ) remained unchanged in average, but significant decrease was observed in responders and significant increase in non-responders. QRS width, SPWMD and Ts-sep-lat showed moderate sensitivity but poor specificity to predict CRT benefit. QRS width $\geq 150 \mathrm{~ms}$ in niCMP patients showed higher sensitivity to predict CRT effect compared to iCMP patients ( $91 \%, 65 \%$ respectively). IVMD showed poor sensitivity but good specificity to predict CRT benefit. IVMD in SR patients (compared to AF patients) showed higher specificity to predict CRT effect (90\%, 63\% respectively).

Conclusion. None of tested left ventricle contraction dyssynchrony parameters showed good sensitivity and specificity to predict CRT benefit. QRS width as a predictor factor was more beneficial in non-ischemic patients and IVMD in sinus rhythm patients.

\section{INTRODUCTION}

Biventricular pacing as a modality of cardiac resynchronization therapy is already a common non-pharmacologic treatment of drug-resistant heart failure in patients with left ventricle contraction dysfunction and left ventricle contraction dyssynchrony (thereinafter dyssynchrony). Contemporary guidelines define "dyssynchrony" as QRS width usually above $120 \mathrm{~ms}^{1-3}$.

Randomized multicenter studies have showed clear benefit of CRT on functional status improvement, reversed remodeling of dilated left ventricle and reduction of total and cardiovascular mortality and hospitalization rate $^{4-9}$. Following the classical indication criteria does not lead to positive response to CRT in $30-40 \%$ of patients ${ }^{10}$. The reason of such a high number of so called "non-re- sponders" is multifactorial - including labile relationship between electrical (QRS width) and mechanical dyssynchrony ${ }^{11}$, influence of other factors such as myocardial ischemia and presence of fibrous tissue, final lead position, concomitant diseases and arrhythmias etc.

\section{AIM}

The aim of our study was 1) to describe the importance of the basal QRS width and basal dyssynchrony parameters assessment for prediction of CRT benefit, 2) to compare benefit of these parameters assessment in patients with ischemic (iCMP) and non-ischemic cardiomyopathy (niCMP) and with sinus rhythm (SR) and atrial fibrillation (AF). 


\section{PATIENTS AND METHODS}

In retrospective study, we analyzed a group of 194 consecutive patients, CRT-P (biventricular pacemaker) and CRT-D (biventricular cardioverter-defibrillators) recipients, implanted from September 2000 to March 2009. Inclusion criteria were successful implantation of CRT device, effective biventricular pacing (defined as effective pacing and proper sensing on both ventricular leads and proper function of atrial lead in patients with sinus rhythm) and ventricular pacing percentage not decreasing bellow $80 \%$ according to device data.

Before implantation of CRT device, following parameters were assessed: NYHA function class, ECG recording with assessment of basal rhythm and complex QRS width, echocardiography with assessment of left ventricle ejection fraction (LVEF), enddiastolic and endsystolic diameter of the left ventricle (LVEDD, LVESD) and assessments of dyssynchrony parameters (SPWMD, Ts-seplat, IVMD) - see Table 1.

The same examinations were performed after 3 months of biventricular pacing.

$\mathrm{AV}$ delay was optimized in patients with sinus rhythm, using our developed and published method ${ }^{12}$.

Responders to CRT were defined by the improvement in NYHA class. Patients with at least one coronary artery with a luminal diameter narrowing $>70 \%$ were designated to "ischemic cardiomyopathy (iCMP)" patients. Others, including patients with cardiomyopathy due to valvular disease, were designated to "non-ischemic CMP (niCMP)" diagnosis.

Statistic calculations were performed on StatSoft Statistica 7.0. commercial software. Comparison between patient subgroups was calculated using Man-Whitney $\mathrm{U}$ test, significance of difference between basal values and values after CRT in continuous parameters was calculated using Wilcoxon match paired test. Statistical significance was set as $5 \%$ level.

\section{RESULTS}

\section{A. Changes in NYHA class and left ventricular function and diameters after CRT implantation}

Tables 2 and 3 contain values of analyzed parameters in the whole group and subgroups of responders and nonresponders (Table 2) and subgroups defined based on heart failure etiology and basal heart rhythm (Table 3).

NYHA class changed from average 2.9 to 2.4 but only $61 \%$ of patients showed NYHA class improvement. There was no difference in responder numbers and NYHA change between patients with iCMP and niCMP. Lower percentage of responders $(52 \%$ vs. $63 \%$, p < 0.05$)$ was in AF patients compared to SR patients.

LVEF, LVEDD and LVESD significantly improved in all subgroups except for non-responders.

\section{B. Change of QRS width and dyssynchrony markers after CRT implantation}

In our group, responders had significantly wider basal QRS width compared to non-responders (166 ms vs. 157 $\mathrm{ms}, \mathrm{p}=0.03)$. Responders showed statistically significant shortening of QRS width (change from $166 \mathrm{~ms}$ to $155 \mathrm{~ms}$, $\mathrm{p}<0.01$ ). On contrary, non-responders showed widening (change from $157 \mathrm{~ms}$ to $165 \mathrm{~ms}, \mathrm{p}=0.02$ ). Narrowing of QRS after CRT was more expressed in patients with niCMP (change from $166 \mathrm{~ms}$ to $162 \mathrm{~ms}, \mathrm{p}=0.02$ ) versus iCMP patients (changed from $155 \mathrm{~ms}$ to $158 \mathrm{~ms}$, NS). But iCMP and niCMP patients differed in basal QRS width significantly $(\mathrm{p}<0.01)$.

Interval SPWMD and Ts-sep-lat significantly decreased after CRT in all subgroups including non-responders. Reduction in percentage of patients with mechanical dyssynchrony defined as SPWMD $\geq 130 \mathrm{~ms}$ (after CRT device implantation) was detectable in all subgroups except for non-responders and iCMP patients. Occurrence of pathological value of Ts-sep-lat was lowered by CRT pacing only in responders and patients with sinus rhythm. Interval IVMD remained unchanged in the whole group but responders presented a significant reduction while non-responders an increase in IVMD value. Percentage of patients with IVMD dyssynchrony was significantly lower in responders and significantly higher in non-responders.

\section{Difference in outcome between patients with versus without dyssynchrony}

The results are summarized in Table 4.

Percentages of responders (with NYHA class improvement) and patients with improvement in individual echocardiographic parameters (LVEF, LVEDD, LVESD) were calculated for situation when CRT candidates are selected according to one dyssynchrony parameter (QRS width, SPWMD, Ts-sep-lat and IVMD) with standard cut-off values. There was no statistical difference in CRT benefit between patients with vs. without dyssynchrony in any analyzed dyssynchrony parameters but some trends were detectable.

Patients with $\mathrm{QRS} \geq 150 \mathrm{~ms}$ had non-significantly better outcome in LVEF improvement ( $55 \%$ vs. $32 \%, p=0.2$ ).

In our group, patients with SPWMD dyssynchrony had worse outcome (in NYHA class and LVEF improvement and LVEDD and LVESD reduction) compared with patients without SPWMD dyssynchrony. Patients with and without Ts-sep-lat dyssynchrony did not differ at all in the outcome.

Patients with IVMD dyssynchrony, compared to those without dyssynchrony, showed better outcome in NYHA class and LVEF improvement but the change was not statistically significant ( $p=0.1$ and $p=0.09$ respectively).

\section{Statistical power of QRS width and dyssynchrony \\ parameters to predict CRT effect}

The results are summarized in Table 5.

QRS width with cut-off value of $150 \mathrm{~ms}$ and SPWMD with cut-off value of $130 \mathrm{~ms}$ had acceptable sensitivities ( 83 and $87 \%$ respectively) and poor specificities (23\% and 
Table 1. Evaluated echocardiography parameters.

\begin{tabular}{|l|l|}
\hline LVEF & $\begin{array}{l}\text { LVEF assessed by biplanar method, from apical 4-chamber and 2-chamber projection, lining of endo- } \\
\text { card contours in systole and diastole } \\
\text { device automatic software. }\end{array}$ \\
\hline $\begin{array}{l}\text { LVEDD } \\
\text { LVEDS }\end{array}$ & $\begin{array}{l}\text { Both values measured in parasternal long axis projection as diameter of left ventricle at the end of } \\
\text { diastole and systole. }\end{array}$ \\
\hline SPWMD $^{15}$ & $\begin{array}{l}\text { Interval responding to time delay between maximum movement of posterior wall and septum (septal- } \\
\text { to-posterior wall motion delay, SPWMD) measured in M-mode echocardiography in parasternal long } \\
\text { axis, in plane just bellow mitral valve at the level of papillary muscles. SPWMD } \geq 130 \text { ms was diagnosed } \\
\text { as "intraventricular mechanical delay" present. }\end{array}$ \\
\hline Ts-sep-lat ${ }^{19}$ & $\begin{array}{l}\text { Interval between peak myocardial velocity in basal septal and basal lateral segment in tissue doppler } \\
\text { imaging (TDI) in apical 4-chamber projection. Cut-off value of dyssynchrony presence is } 65 \text { ms. }\end{array}$ \\
\hline IVMD 25 & $\begin{array}{l}\text { Interventricular mechanical delay, calculated as difference between left preejection interval (LPEI) and } \\
\text { right preejection interval (RPEI). LPEI is measured by PW Doppler in apical long axis projection and } \\
\text { RPEI in short axis parasternal projection with sample volume in level of aortic (LPEI) or pulmonary } \\
\text { (RPEI) valve. LPEI and RPEI is the interval from QRS beginning to start of ejection flow. Cut-off } \\
\text { value of dysynchrony presence is } 60 \text { ms. }\end{array}$ \\
\hline
\end{tabular}

$5 \%$ respectively) for CRT benefit prediction. QRS width in niCMP subgroup had better sensitivity (91\%). Ts-sep-lat had moderate sensitivity $(71 \%)$ and poor specificity $(23 \%$, in iCMP subgroup 30\%) for CRT benefit prediction.

IVMD differed from above mentioned markers. The sensitivity for CRT benefit prediction was poor ( $56 \%$, even worse in iCMP subgroup - 42\%) but the specificity was much better $(82 \%, 90 \%$ in SR group vs. $63 \%$ in $\mathrm{AF}$ group).

\section{DISSCUSSION}

Cotemporary guidelines for CRT device implantation include depression of left ventricle systolic function with $\mathrm{LVEF} \leq 35 \%$ and $\mathrm{QRS}$ width $\geq 120 \mathrm{~ms}^{1-3}$. Morphology of QRS is not specified ${ }^{1-2}$ and mechanical dyssynchrony demonstration is not needed to be proven.

QRS width presents the amount of electrical dyssynchrony. Many studies proved that electrical dyssynchrony did not correlate with mechanical dyssynchrony ${ }^{13-15}$. However, mechanical dyssynchrony can be caused by several reasons:

1. electrical conduction disorder in left ventricle (left bundle branch block). Only this is treatable by CRT.

2. structural changes in left ventricle segments (presence of fibrous tissue $)^{16}$

3. myocardial ischemia ${ }^{17}$

Several methods for dyssynchrony assessment were published but no one is optimal. Nowadays, parameters based on tissue Doppler imaging are popular. These parameters reflect myocardial velocity and do not differentiate passive movement and active contraction. Parameters based on deformation detection are more perspective but very sensitive on artifacts.

SPWMD was published by Pitzalis et al..$^{15}$ as early as 2002 and was tested only for non-ischemic cardiomyopathy. We found no differences between iCMP and niCMP.
Concordantly with author, we observed good sensitivity but incomparably worse specificity (63 vs. $5 \%$ ) to predict NYHA class improvement after CRT. Controlled THE PROSPECT Study showed no difference in basal SPWMD between responders versus non-responders but showed significant difference $(\mathrm{p}=0.021)$ between patients with vs. without left ventricle endsystolic volume (LVESV) reduction after $\mathrm{CRT}^{18}$. In our group of patients, basal SPWMD did not differ between responders and non-responders $(p=0.17)$ in concordance with THE PROSPECT Study, but we proved no difference between patients with vs. without LVEF, LVEDD or LVESD reduction after CRT ( $p=0.7, p=0.13$ and $p=0.08$ respectively). Our patients with SPWMD dyssynchrony had even worse outcome in NYHA improvement than patients without dyssynchrony. It was probably caused by very low number of patients with SPWMD bellow $130 \mathrm{~ms}$ in our analysis.

Ts-sep-lat is the easiest parameter based on tissue Doppler imaging. On group of 25 people, Bax et al. ${ }^{19}$ demonstrated good power of Ts-sep-lat to predict CRT benefit. In our group $(n=71)$, it presented neither acceptable sensitivity nor specificity in NYHA class improvement and echocardiographic parameters improvement prediction. Controlled THE PROSPECT Study showed no significant differences in Ts-sep-lat between responders versus non-responders but significant difference between patients with vs. without LVESV reduction $(n=0.005)$. In our group, significant difference between responders vs. non-responders $(p=0.05)$ and between patients with vs. without LVEF improvement ( $p=0.05)$ was observed. We found no difference in basal Ts-sep-lat between patients with vs. without LVEDD or LVESD reduction after CRT.

QRS width, SPWMD and Ts-sep-lat showed acceptable sensitivity but poor specificity for CRT benefit prediction. Following one of these markers (with defined cut-off values) theoretically would indicate to CRT relatively high percentage of patients that would really profit from CRT, but would produce a high amount of non-responders. 
Table 2. Demographic data and values of NYHA class, QRS width and echocardiographic parameters in the whole group and subgroups of NYHA responders and non-responders.

\begin{tabular}{|c|c|c|c|c|}
\hline & \multirow{2}{*}{ All patients } & \multicolumn{3}{|c|}{ NYHA response 3 months after CRT implantation } \\
\hline & & NYHA responders & $\begin{array}{c}\text { NYHA } \\
\text { non-responders }\end{array}$ & $\mathrm{P}$ \\
\hline $\begin{array}{l}\text { Number of pts [N] } \\
\text { Males [\%] } \\
\text { Age [years] } \\
\text { Sinus rhythm [\%] } \\
\text { Non-ischemic CMP [\%] }\end{array}$ & $\begin{array}{c}194 \\
75 \% \\
62.1 \pm 9.4 \\
78 \% \\
69 \% \\
\end{array}$ & $\begin{array}{c}119(61 \%) \\
72 \% \\
61.6 \pm 8.9 \\
82 \% \\
69 \% \\
\end{array}$ & $\begin{array}{c}75(39 \%) \\
79 \% \\
62.9 \pm 10.1 \\
73 \% \\
68 \% \\
\end{array}$ & \\
\hline $\begin{array}{l}\text { NYHA class } \\
\text { - before CRT } \\
\text { - after CRT } \\
\text { - improved by } 1 / 2 \text { class [\% pts] }\end{array}$ & $\begin{array}{c}2.9 \pm 0.3 \\
2.4 \pm 0.5^{* * *} \\
61 \%\end{array}$ & $\begin{array}{c}3.0 \pm 0.3 \\
2.1 \pm 0.4^{* * *} \\
100 \%\end{array}$ & $\begin{array}{c}2.7 \pm 0.3 \\
2.8 \pm 0.4 \text { * } \\
0 \%\end{array}$ & $\begin{array}{l}\$ \$ \$ \\
\$ \$ \$ \\
\$ \$ \$\end{array}$ \\
\hline $\begin{array}{l}\text { QRS width [ms] } \\
\text { - before CRT } \\
\text { - after CRT } \\
\text { - QRS shortened [\% pts] }\end{array}$ & $\begin{array}{c}163 \pm 23 \\
161 \pm 21 \\
49 \%\end{array}$ & $\begin{array}{c}166 \pm 23 \\
155 \pm 21^{* * *} \\
58 \%\end{array}$ & $\begin{array}{c}157 \pm 20 \\
165 \pm 21 \text { * } \\
34 \%\end{array}$ & $\begin{array}{c}\$ \$ \\
\$ \\
\$ \$\end{array}$ \\
\hline $\begin{array}{l}\text { LVEF [\%] } \\
\text { - before CRT } \\
\text { - after CRT } \\
\text { - improved by } \geq 10 \%[\% \mathrm{pts}]\end{array}$ & $\begin{array}{c}21.3 \pm 5.8 \\
24.0 \pm 6.5^{* * *} \\
52 \%\end{array}$ & $\begin{array}{c}20.8 \pm 5.4 \\
24.8 \pm 6.5^{* * *} \\
63 \%\end{array}$ & $\begin{array}{c}21.9 \pm 6.4 \\
22.7 \pm 6.3 \\
33 \% \\
\end{array}$ & $\$$ \\
\hline $\begin{array}{l}\text { LVEDD [mm] } \\
\text { - before CRT } \\
\text { - after CRT } \\
\text { - improved by } \geq 5 \%[\% \mathrm{pts}]\end{array}$ & $\begin{array}{c}68 \pm 9 \\
67 \pm 10^{* * *} \\
33 \% \\
\end{array}$ & $\begin{array}{c}69 \pm 9 \\
67 \pm 10^{* * *} \\
36 \% \\
\end{array}$ & $\begin{array}{c}67 \pm 9 \\
67 \pm 9 \\
26 \% \\
\end{array}$ & \\
\hline $\begin{array}{l}\text { LVESD }[\mathrm{mm}] \\
\text { - before CRT } \\
\text { - after CRT } \\
\text { - improved by } \geq 5 \%[\% \mathrm{pts}]\end{array}$ & $\begin{array}{c}61 \pm 11 \\
58 \pm 12^{* * *} \\
49 \%\end{array}$ & $\begin{array}{c}62 \pm 11 \\
57 \pm 12^{* * *} \\
59 \% \\
\end{array}$ & $\begin{array}{c}60 \pm 12 \\
60 \pm 11 \\
30 \% \\
\end{array}$ & $\$ \$ \$$ \\
\hline $\begin{array}{l}\text { SPWMD [ms] } \\
\text { - before CRT } \\
\text { - after CRT } \\
\text { - dyssynchrony before [\% pts] } \\
\text { - dyssynchrony after [\% pts] }\end{array}$ & $\begin{array}{c}210 \pm 84 \\
140 \pm 59^{* * *} \\
86 \% \\
60 \%{ }^{* * *} \\
\end{array}$ & $\begin{array}{c}202 \pm 81 \\
124 \pm 45^{* * *} \\
87 \% \\
51 \%^{* * *} \\
\end{array}$ & $\begin{array}{c}217 \pm 84 \\
165 \pm 71^{* * *} \\
83 \% \\
72 \% \\
\end{array}$ & $\begin{array}{l}\$ \$ \$ \\
\$ \$\end{array}$ \\
\hline $\begin{array}{l}\text { Ts-sep-lat [ms] } \\
\text { - before CRT } \\
\text { - after CRT } \\
\text { - dyssynchrony before [\% pts] } \\
\text { - dyssynchrony after [\% pts] }\end{array}$ & $\begin{array}{c}92 \pm 53 \\
72 \pm 32^{* * *} \\
73 \% \\
54 \%^{*} \\
\end{array}$ & $\begin{array}{c}83 \pm 43 \\
61 \pm 24^{* * *} \\
71 \% \\
44 \% * \\
\end{array}$ & $\begin{array}{c}110 \pm 65 \\
92 \pm 37 \\
80 \% \\
72 \%\end{array}$ & $\begin{array}{c}\$ \\
\$ \$ \$ \\
\$\end{array}$ \\
\hline $\begin{array}{l}\text { IVMD } \\
\text { - before CRT } \\
\text { - after CRT } \\
\text { - dyssynchrony before [\% pts] } \\
\text { - dyssynchrony after [\% pts] }\end{array}$ & $\begin{array}{c}52 \pm 35 \\
51 \pm 26 \\
41 \% \\
40 \%\end{array}$ & $\begin{array}{c}58 \pm 34 \\
50 \pm 26^{*} \\
56 \% \\
34 \%^{*}\end{array}$ & $\begin{array}{c}42 \pm 31 \\
53 \pm 36 \text { ** } \\
18 \% \\
46 \%{ }^{*}\end{array}$ & $\$$ \\
\hline
\end{tabular}

Continuous values presented as average \pm standard deviation. Frequency of occurrence presented as $\%$ of patients in individual subgroups. Improvement in LVEF, LVEDD a LVESD parameters was positive when $5 \%$ or $10 \%$ improvement of basal value was presented. $\mathrm{P}$ = statistical value

Statistical significance signed using these symbols:

* change between values before and after CRT at $p<5 \%$ statistical level

** change between values before and after CRT at $\mathrm{p}<1 \%$ statistical level

${ }^{* * * *}$ change between values before and after CRT at $\mathrm{p}<0.1 \%$ statistical level

$\$$ change between subgroups of patients at $p<5 \%$ statistical level

$\$$ change between subgroups of patients at $p<1 \%$ statistical level

$\$ \$$ change between subgroups of patients at $p<0.1 \%$ statistical level 
Table 3. Demographic data and values of NYHA class, QRS width and echocardiographic parameters in subgroups divided according to heart failure etiology and basal rhythm.

\begin{tabular}{|c|c|c|c|c|c|c|}
\hline & \multicolumn{3}{|c|}{ Heart failure etiology } & \multicolumn{3}{|c|}{ Basal rhythm } \\
\hline & niCMP & iCMP & $\mathrm{P}$ & SR & $\mathrm{AF}$ & $\mathrm{P}$ \\
\hline $\begin{array}{l}\text { Number of pts [N] } \\
\text { Males [\%] } \\
\text { Age [years] } \\
\text { Sinus rhythm [\%] } \\
\text { Non-ischemic CMP [\%] }\end{array}$ & $\begin{array}{c}133 \\
74 \% \\
60.2 \pm 9.4 \\
78 \% \\
100 \% \\
\end{array}$ & $\begin{array}{c}61 \\
77 \% \\
66.2 \pm 7.9 \\
79 \% \\
0 \% \\
\end{array}$ & $\begin{array}{l}\$ \$ \$ \\
\$ \$ \$\end{array}$ & $\begin{array}{c}152 \\
74 \% \\
61.5 \pm 9.4 \\
100 \% \\
68 \% \\
\end{array}$ & $\begin{array}{c}42 \\
76 \% \\
64.4 \pm 9.1 \\
0 \% \\
69 \% \\
\end{array}$ & $\$ \$$ \\
\hline $\begin{array}{l}\text { NYHA class } \\
\text { - before CRT } \\
\text { - after CRT } \\
\text { - improved by } 1 / 2 \text { class [ } \% \text { pts] }\end{array}$ & $\begin{array}{c}2.9 \pm 0.3 \\
2.4 \pm 0.5^{* * *} \\
62 \%\end{array}$ & $\begin{array}{c}2.9 \pm 0.3 \\
2.4 \pm 0.5^{* * *} \\
61 \%\end{array}$ & & $\begin{array}{c}2.8 \pm 0.3 \\
2.4 \pm 0.5^{* * *} \\
63 \%\end{array}$ & $\begin{array}{c}2.9 \pm 0.3 \\
2.5 \pm 0.4^{* * *} \\
52 \%\end{array}$ & $\begin{array}{l}\$ \\
\$\end{array}$ \\
\hline $\begin{array}{l}\text { QRS width [ms] } \\
\text { - before CRT } \\
\text { - after CRT } \\
\text { - QRS shortened [\% pts] }\end{array}$ & $\begin{array}{c}166 \pm 22 \\
162 \pm 20 \text { * } \\
53 \%\end{array}$ & $\begin{array}{c}155 \pm 23 \\
158 \pm 21 \\
41 \% \\
\end{array}$ & $\$ \$$ & $\begin{array}{c}163 \pm 23 \\
160 \pm 20 \\
47 \% \\
\end{array}$ & $\begin{array}{c}163 \pm 23 \\
163 \pm 25 \\
54 \%\end{array}$ & \\
\hline $\begin{array}{l}\text { LVEF [\%] } \\
\text { - before CRT } \\
\text { - after CRT } \\
\text { - improved by } \geq 10 \%[\% \mathrm{pts}]\end{array}$ & $\begin{array}{c}20.6 \pm 5.8 \\
23.7 \pm 6.7^{* * * *} \\
55 \%\end{array}$ & $\begin{array}{c}22.7 \pm 5.4 \\
24.6 \pm 6.5^{* * *} \\
55 \% \\
\end{array}$ & $\$$ & $\begin{array}{c}21.2 \pm 5.7 \\
23.9 \pm 6.3^{* * *} \\
53 \%\end{array}$ & $\begin{array}{c}21.5 \pm 6.2 \\
24.5 \pm 7.0 \text { ** } \\
47 \%\end{array}$ & \\
\hline $\begin{array}{l}\text { LVEDD [mm] } \\
\text { - before CRT } \\
\text { - after CRT } \\
\text { - improved by } \geq 5 \%[\% \mathrm{pts}]\end{array}$ & $\begin{array}{c}69 \pm 9 \\
67 \pm 10 \text { ** } \\
35 \% \\
\end{array}$ & $\begin{array}{c}68 \pm 9 \\
67 \pm 8^{*} \\
28 \%{ }^{(*)}\end{array}$ & & $\begin{array}{c}69 \pm 10 \\
67 \pm 10 \text { ** } \\
31 \%\end{array}$ & $\begin{array}{c}68 \pm 7 \\
65 \pm 8 * \\
39 \%\end{array}$ & \\
\hline $\begin{array}{l}\text { LVESD }[\mathrm{mm}] \\
\text { - before CRT } \\
\text { - after CRT } \\
\text { - improved by } \geq 5 \%[\% \mathrm{pts}]\end{array}$ & $\begin{array}{c}62 \pm 11 \\
58 \pm 12 \\
50 \% \\
\end{array}$ & $\begin{array}{c}60 \pm 11 \\
58 \pm 10^{* *} \\
47 \%\end{array}$ & & $\begin{array}{c}62 \pm 12 \\
58 \pm 12^{* * * *} \\
46 \% \\
\end{array}$ & $\begin{array}{c}60 \pm 9 \\
56 \pm 11^{* *} \\
58 \% \\
\end{array}$ & \\
\hline $\begin{array}{l}\text { SPWMD [ms] } \\
\text { - before CRT } \\
\text { - after CRT } \\
\text { - dyssynchrony before [\% pts] } \\
\text { - dyssynchrony after [\% pts] }\end{array}$ & $\begin{array}{c}216 \pm 89 \\
140 \pm 65^{* * *} \\
91 \% \\
57 \% \text { * }\end{array}$ & $\begin{array}{c}195 \pm 70 \\
140 \pm 44^{* * *} \\
86 \% \\
65 \% \\
\end{array}$ & & $\begin{array}{c}215 \pm 84 \\
145 \pm 59^{* * *} \\
92 \% \\
63 \%{ }^{* * *} \\
\end{array}$ & $\begin{array}{c}190 \pm 82 \\
117 \pm 59^{* * *} \\
79 \% \\
46 \%{ }^{*}\end{array}$ & $\$$ \\
\hline $\begin{array}{l}\text { Ts-sep-lat [ms] } \\
\text { - before CRT } \\
\text { - after CRT } \\
\text { - dyssynchrony before [\% pts] } \\
\text { - dyssynchrony after [\% pts] }\end{array}$ & $\begin{array}{c}91 \pm 48 \\
72 \pm 33^{* * *} \\
75 \% \\
55 \% \\
\end{array}$ & $\begin{array}{c}95 \pm 64 \\
71 \pm 33^{* *} \\
70 \% \\
52 \% \\
\end{array}$ & & $\begin{array}{c}96 \pm 57 \\
74 \pm 35^{\text {**** }} \\
75 \% \\
56 \% \text { ** } \\
\end{array}$ & $\begin{array}{c}79 \pm 32 \\
65 \pm 20^{*} \\
67 \% \\
44 \%\end{array}$ & \\
\hline $\begin{array}{l}\text { IVMD } \\
\text { - before CRT } \\
\text { - after CRT } \\
\text { - dyssynchrony before [\% pts] } \\
\text { - dyssynchrony after [\% pts] }\end{array}$ & $\begin{array}{c}55 \pm 34 \\
54 \pm 23 \\
46 \% \\
41 \%\end{array}$ & $\begin{array}{c}48 \pm 37 \\
47 \pm 30 \\
33 \% \\
37 \%\end{array}$ & & $\begin{array}{c}49 \pm 33 \\
48 \pm 26 \\
41 \% \\
37 \%\end{array}$ & $\begin{array}{c}62 \pm 40 \\
58 \pm 25 \\
41 \% \\
47 \%\end{array}$ & \\
\hline
\end{tabular}

Legend identical with Table 2

niCMP = non-ischemic cardiomyopathy, iCMP = ischemic cardiomyopathy, $\mathrm{SR}=$ sinus rhythm,

$\mathrm{AF}=$ atrial fibrillation, $\mathrm{P}=$ statistical significance

IVMD is a parameter reflecting interventricular dyssynchrony. It could by influenced by many factors. Even though, the PROSPECT study showed significant difference $(p=0.045)$ in basal IVMD between responders vs. non-responders and significant difference $(p=0.029)$ between patients with vs. without LVESV reduction after CRT. Similar results were observed in SCART trial ${ }^{20}$. In our group, we observed difference in basal IVMD between responders vs. non-responders $(\mathrm{p}=0.02)$ and patients with vs. without LVEF reduction $(p=0.01)$ after CRT. Patients with vs. without LVEDD or LVESD reduction after CRT did not differ in basal IVMD in our group. IVMD showed high specificity (especially in SR patients) but poor sensitivity in CRT benefit prediction. This parameter (being the only criterion) would theoretically refuse a high amount of suitable patients to implantation but (only in SR patients) would produce only around $10 \%$ of non-responders. 
Table 4. Percentage of patients with improvement in NYHA class and in echocardiographic parameters after CRT in dependence on presence of basal electrical and mechanical dyssynchrony.

\begin{tabular}{|c|c|c|c|c|}
\hline Patients subgroups & $\begin{array}{c}\% \text { of pts } \\
\text { with improve- } \\
\text { ment in NYHA }\end{array}$ & $\begin{array}{c}\% \text { of pts } \\
\text { with improvement } \\
\text { in LVEDD }\end{array}$ & $\begin{array}{c}\% \text { of pts } \\
\text { with improvement } \\
\text { in LVESD }\end{array}$ & $\begin{array}{c}\% \text { of pts } \\
\text { with improvement } \\
\text { in EF }\end{array}$ \\
\hline $\begin{array}{l}\text { QRS width }<150 \mathrm{~ms} \\
(\mathrm{n}=34)\end{array}$ & $53 \%$ & $26 \%$ & $41 \%$ & $32 \%$ \\
\hline $\begin{array}{l}\text { QRS width } \geq 150 \mathrm{~ms} \\
(\mathrm{n}=145)\end{array}$ & $63 \%$ & $34 \%$ & $50 \%$ & $55 \%$ \\
\hline Significance & $\mathrm{p}=0.6$ & $\mathrm{p}=0.5$ & $\mathrm{p}=0.6$ & $\mathrm{p}=0.2$ \\
\hline SPWMD $<130 \mathrm{~ms}(\mathrm{n}=14)$ & $79 \%$ & $64 \%$ & $64 \%$ & $79 \%$ \\
\hline $\begin{array}{l}\text { SPWMD } \geq 130 \mathrm{~ms} \\
(\mathrm{n}=123)\end{array}$ & $58 \%$ & $27 \%$ & $44 \%$ & $50 \%$ \\
\hline Significance & $\mathrm{p}=0.5$ & $\mathrm{p}=0.07$ & $\mathrm{p}=0.4$ & $\mathrm{p}=0.3$ \\
\hline Ts-sep-lat < $65 \mathrm{~ms}(\mathrm{n}=19)$ & $68 \%$ & $26 \%$ & $42 \%$ & $58 \%$ \\
\hline Ts-sep-lat $\geq 65 \mathrm{~ms}(\mathrm{n}=52)$ & $62 \%$ & $35 \%$ & $46 \%$ & $42 \%$ \\
\hline Significance & $\mathrm{p}=0.8$ & $p=0.6$ & $\mathrm{p}=0.8$ & $\mathrm{p}=0.5$ \\
\hline IVMD < $60 \mathrm{~ms}(\mathrm{n}=42)$ & $45 \%$ & $33 \%$ & $48 \%$ & $38 \%$ \\
\hline IVMD $\geq 60 \mathrm{~ms}(\mathrm{n}=29)$ & $82 \%$ & $24 \%$ & $48 \%$ & $76 \%$ \\
\hline Significance & $\mathrm{p}=0.1$ & $\mathrm{p}=0.5$ & $\mathrm{p}=1.0$ & $\mathrm{p}=0.09$ \\
\hline
\end{tabular}

Table 5. Sensitivity, specificity and predictive values for CRT response prediction in situation the indication criterion is set according to individual parameter of electrical or mechanical dyssynchrony.

\begin{tabular}{|c|c|c|c|c|c|}
\hline $\begin{array}{l}\text { Parameter of dyssynchrony } \\
\text { and cut-off value } \\
\text { - tested subgroups }\end{array}$ & $\mathrm{N}$ & Sensitivity & Specificity & $\begin{array}{l}\text { Positive } \\
\text { predictive } \\
\text { value }\end{array}$ & $\begin{array}{l}\text { Negative } \\
\text { predictive } \\
\text { value }\end{array}$ \\
\hline $\begin{array}{l}\text { QRS width } \geq 150 \mathrm{~ms} \\
\text { - in all pts } \\
\text { - in niCMP / iCMP pts } \\
\text { - in SR / AF pts } \\
\text { - in niCMP+SR / niCMP+AF pts } \\
\text { - in iCMP+SR / iCMP+AF pts }\end{array}$ & $\begin{array}{c}149 \\
111 / 38 \\
122 \text { / } 27 \\
91 / 20 \\
31 / 7 \\
\end{array}$ & $\begin{array}{c}83 \% \\
91 / 65 \% \\
85 / 76 \% \\
91 / \text { NE\% } \\
68 \text { / NE\% }\end{array}$ & $\begin{array}{c}23 \% \\
20 / 30 \% \\
19 / 39 \% \\
14 / \text { NE\% } \\
26 / \text { NE\% }\end{array}$ & $\begin{array}{c}63 \% \\
65 \text { / 58\% } \\
64 \text { / 59\% } \\
67 \text { / NE\% } \\
55 \text { / NE\% }\end{array}$ & $\begin{array}{c}47 \% \\
59 \text { / } 37 \% \\
42 \text { / } 58 \% \\
43 \text { / NE\% } \\
38 \text { / NE\% } \\
\end{array}$ \\
\hline $\begin{array}{l}\text { SPWMD } \geq 130 \mathrm{~ms} \\
\text { - in all pts } \\
\text { - in niCMP / iCMP pts } \\
\text { - in SR / AF pts } \\
\text { - in niCMP+SR / niCMP+AF pts } \\
\text { - in iCMP+SR / iCMP+AF pts } \\
\end{array}$ & $\begin{array}{c}123 \\
86 / 37 \\
104 \text { / } 19 \\
71 / 15 \\
33 \text { / } 4 \\
\end{array}$ & $\begin{array}{c}87 \% \\
89 \text { / 81\% } \\
90 \text { / NE\% } \\
90 \text { / NE\% } \\
\text { NE / NE }\end{array}$ & $\begin{array}{c}5 \% \\
5 / 6 \% \\
4 / \mathrm{NE} \% \\
7 / \mathrm{NE} \% \\
\mathrm{NE} / \mathrm{NE} \\
\end{array}$ & $\begin{array}{c}58 \% \\
58 / 57 \% \\
62 / \mathrm{NE} \% \\
62 / \mathrm{NE} \% \\
\mathrm{NE} / \mathrm{NE} \\
\end{array}$ & $\begin{array}{c}21 \% \\
25 / 17 \% \\
22 / \mathrm{NE} \% \\
29 \text { / NE\% } \\
\text { NE / NE }\end{array}$ \\
\hline $\begin{array}{l}\text { Ts-sep-lat } \geq 65 \mathrm{~ms} \\
\text { - in all pts } \\
\text { - in niCMP / iCMP pts } \\
\text { - in SR / AF pts } \\
\text { - in niCMP+SR / niCMP+AF pts } \\
\text { - in iCMP+SR / iCMP+AF pts }\end{array}$ & $\begin{array}{c}52 \\
33 / 19 \\
41 / 11 \\
25 / 8 \\
26 / 3 \\
\end{array}$ & $\begin{array}{c}71 \% \\
71 / 71 \% \\
70 / \mathrm{NE} \% \\
\mathrm{NE} / \mathrm{NE} \\
\mathrm{NE} / \mathrm{NE} \\
\end{array}$ & $\begin{array}{c}23 \% \\
19 / 30 \% \\
17 \text { / NE\% } \\
\text { NE / NE } \\
\text { NE / NE }\end{array}$ & $\begin{array}{c}62 \% \\
61 / 63 \% \\
63 / \mathrm{NE} \% \\
\mathrm{NE} / \mathrm{NE} \\
\mathrm{NE} / \mathrm{NE} \\
\end{array}$ & $\begin{array}{c}32 \% \\
27 / 38 \% \\
21 / \mathrm{NE} \% \\
\mathrm{NE} / \mathrm{NE} \\
\mathrm{NE} / \mathrm{NE}\end{array}$ \\
\hline $\begin{array}{l}\text { IVMD } \geq 60 \mathrm{~ms} \\
\text { - in all pts } \\
\text { - in niCMP / iCMP pts } \\
\text { - in SR / AF pts } \\
\text { - in niCMP+SR / niCMP+AF pts } \\
\text { - in iCMP+SR / iCMP+AF pts }\end{array}$ & $\begin{array}{c}29 \\
19 / 10 \\
22 / 7 \\
13 / 6 \\
9 / 1\end{array}$ & $\begin{array}{c}56 \% \\
66 / 42 \% \\
59 / 44 \% \\
\text { NE / NE } \\
\text { NE / NE }\end{array}$ & $\begin{array}{c}82 \% \\
82 / 82 \% \\
90 / 63 \% \\
\text { NE / NE } \\
\text { NE / NE }\end{array}$ & $\begin{array}{c}83 \% \\
84 / 80 \% \\
91 / 57 \% \\
\text { NE / NE } \\
\text { NE / NE }\end{array}$ & $\begin{array}{c}55 \% \\
64 / 45 \% \\
56 / 50 \% \\
\text { NE / NE } \\
\text { NE / NE }\end{array}$ \\
\hline
\end{tabular}

pts = patients, niCMP = non-ischemic CMP, iCMP $=$ ischemic $\mathrm{CMP}, \mathrm{SR}=$ sinus rhythm, $\mathrm{AF}=$ atrial fibrillation, $\mathrm{NE}=$ not evaluated due to number of analyzed patients bellow 7 (in dyssynchrony or non-dyssynchrony subgroup) Sensitivity $=$ percentage of pts with potential to benefit from CRT that are really approved to CRT Specificity $=$ percentage of pts without potential to benefit from CRT that are really refused to CRT Positive predictive value $=$ percentage of pts approved to CRT who really will be responders Negative predictive value $=$ percentage of pts refused to CRT who really would be non-responders 
It is highly probable that isolated information about mechanical or electrical dyssynchrony is insufficient ${ }^{21}$ for predicting of CRT effect. There is a strong need for information about coronary vessels, presence of ischemia, myocardial viability, optimally using magnetic resonance imaging 22 .

\section{STUDY LIMITATION}

There were several limitations in our study.

Majority of our patients with iCMP had severe coronarography finding, not suitable for intervention. It is probable that ischemic myocardium could mimic a part of dyssynchrony that is logically incapable of resynchronization by CRT device. Even patients after myocardial infarction with myocardium scar were included. Neither dobutamine echocardiography nor fibrous tissue detection was routinely used.

We evaluated parameters that were measured in majority of our patients. We used parameters formerly popular (before the PROSPECT Study publication). Our parameters were relatively easy to examine, not time-consuming, but reflecting real mechanical dyssynchrony only indirectly (IVMD) or only partially (SPWMD, Ts-sep-lat). Measurement of these parameters (especially SPWMD and Ts-sep-lat) is imprecise with low reproducibility and high inter- and intra-observer variability, as we know from the PROSPECT Study ${ }^{18}$.

Rate of dyssynchrony parameters measurement success differed. SPWMD was examined in 182 patients. Segmental left ventricle dysfunction or suboptimal image quality led to impossibility to measure SPWMD in 33 patients. Ts-sep-lat was examined in 122 patients. Unsuccessful measurement due to bad image quality, multi-peak or flat curve of myocardial velocity was in 36 patients. IVMD was examined in 82 patients. Unsuccessful measurement occurred in 7 patients, due to bad quality image of pulmonary flow.

QRS width measurement was evaluated by ECG ruler manually. ECG was recorded at a speed of $25 \mathrm{~mm} / \mathrm{s}$. Values were rounded with 5-10 ms step. We did not separate QRS morphology for analysis because only 4 patients presented right bundle branch block.

NYHA class evaluation is subjective. CRT device implantation could have placebo effect. NYHA improvement in MIRACLE ICD Trial ${ }^{23}$ was present in $63 \%$ with CRT on and in $47 \%$ with CRT off.

\section{CONCLUSION}

We observed NYHA class improvement, LVEF increase and LVEDD and LVESD reduction after CRT device implantation. Responder rate (improvement in NYHA class) was $61 \%$. In AF patient group, the responder rate was significantly worse compared to SR patients.

Significant narrowing of QRS width after CRT was observed only in responders and niCMP patients. CRT reduced SPMWD dyssynchrony more in niCMP patients compared to iCMP patients. Ts-sep-lat reduction was more expressed in SR patients compared to AF patients. IVMD decrease by CRT pacing was observed only in responders.

Responders and non-responders differed in basal QRS width, Ts-sep-lat and IVMD significantly but none of examined (neither mechanical nor electrical) dyssynchrony parameters showed acceptable sensitivity and specificity for CRT benefit prediction. QRS width with cut-off value of $150 \mathrm{~ms}$ showed higher sensitivity for CRT effect prediction in niCMP compared to iCMP patients. IVMD with cut-off value of $60 \mathrm{~ms}$ showed higher specificity for CRT effect prediction in SR patients.

We observed no isolated good predictor of CRT benefit.

\section{ACKNOWLEDGEMENT}

This work was suppoted by grant MSMT 0021622402.

\section{REFERENCES}

1. Pracovní skupina Arytmie a trvalá kardiostimulace České kardiologické společnosti. Zásady pro implantace kadiostimulátorů, implantabilních kardioverterů-defibrilátorů a systémů pro srdeční resynchronizační léčbu 2009. Available from: www.kardio-cz.cz

2. Špinar J, Hradec J, Špác J et al. Doporučení pro diagnostiku a léčbu chronického srdečního selhání ČKS 2006. Suppl Cor Vasa 2007; 49:75-104.

3. Vardas PE, Auricchio A, Blanc JJ et al. Guidelines for cardiac pacing and cardiac resynchronization therapy. The Task Force for Cardiac Pacing and Cardiac Resynchronization Therapy of the European Society of Cardiology. Developed in collaboration with the European Heart Rhythm Association. Europace 2007; 9:95998.

4. Auricchio A, Stellbrink C, Sack S et al. Long-term clinical effect of hemodynamically optimized cardiac resynchronization therapy in patients with heart failure and ventricular conduction delay. J Am Coll Cardiol 2002; 39:2026-33.

5. Cazeau S, Leclercq C, Lavergne $\mathrm{T}$ et al. Effects of multisite biventricular pacing in patients with heart failure and intraventricular conduction delay. N Engl J Med 200; 344:873-80.

6. Gras D, Leclercq C, Tang AS et al. Cardiac resynchronization therapy in advanced heart failure the multicenter InSync clinical study. Eur J Heart Fail 2002; 4:311-20.

7. Abraham WT, Fisher WG, Smith AL et al. Multicenter InSync Randomized Clinical Evaluation. Cardiac resynchronization in chronic heart failure. N Engl J Med. 2002; 346:1845-53.

8. Bristow MR, Saxon LA, Boehmer J et al. Cardiac-resynchronization therapy with or without an implantable defibrillator in advanced chronic heart failure. N Engl J Med 2004; 350:2140-50.

9. Cleland JG, Daubert JC, Erdmann E et al. The effect of cardiac resynchronization therapy on morbidity and mortality in heart failure (the CArdiac REsynchronization-Heart Failure [CARE-HF] Trial). N Engl J Med 2005; 352:1539-49.

10. Bax JJ, Bleeker GB, Marwick TH et al. Left ventricular dyssynchrony predicts response and prognosis after cardiac resynchronization therapy. J Am Coll Cardiol 2004; 44:1834-40.

11. Yu CM, Lin H, Zhang Q et al. High prevalence of left ventricular systolic and diastolic asynchrony in patients with congestive heart failure and normal QRS duration. Heart 2003; 89:54-60.

12. Meluzín J, Novák M, Mullerová J et al. A fast and simple echocardiographic method of determination of the optimal atrioventricular delay in patients after biventricular stimulation. Pacing Clin Electrophysiol 2004; 27:58-64. 
13. Yu CM, Lin H, Zhang Q et al. High prevalence of left ventricular systolic and diastolic asynchrony in patients with congestive heart failure and normal QRS duration. Heart 2003; 89:54-60.

14. Sade LE, Kanzaki H, Severyn D et al. Quantification of radial mechanical dyssynchrony in patients with left bundle branch block and idiopathic dilated cardiomyopathy without conduction delay by tissue displacement imaging. Am J Cardiol 2004; 94:514-18.

15. Pitzalis MV, Iacoviello M, Romito R et al. Ventricular asynchrony predicts a better outcome in patients with chronic heart failure receiving cardiac resynchronization therapy. J Am Coll Cardiol 2005; 45:65-69.

16. Weidemann F, Niemann M, Herrmann S et al. A new echocardiographic approach for the detection of non-ischaemic fibrosis in hypertrophic myocardium. Eur Heart J 2007; 28:3020-26.

17. Winter R, Jussila R, Nowak J et al. Speckle tracking echocardiography is a sensitive tool for the detection of myocardial ischemia: a pilot study from the catheterization laboratory during percutaneous coronary intervention. J Am Soc Echocardiogr 2007; 20:974-81.

18. Chung ES, Leon AR, Tavazzi L et al. Results of the Predictors of Response to CRT (THE PROSPECT) trial. Circulation 2008; 117:2608-16.
19. Bax JJ, Marwick TH, Molhoek SG et al. Left ventricular dyssynchrony predicts benefit of cardiac resynchronization therapy in patients with end-stage heart failure before pacemaker implantation. Am J Cardiol 2003; 92:1238-40.

20. Peraldo C, Achilli A, Orazi S et al. Results of the SCART study: selection of candidates for cardiac resynchronisation therapy. $\mathrm{J}$ Cardiovasc Med (Hagerstown) 2007; 8(11):889-95.

21. Beshai JF, Khunnawat C, Lin AC. Mechanical dyssynchrony from the perspective of a cardiac electrophysiologist. Curr Opin Cardiol 2008; 23:447-51.

22. Abraham J, Abraham TP. Is echocardiographic assessment of dyssynchrony useful to select candidates for cardiac resynchronization therapy? Circ Cardiovasc Imaging 2008; 1:79-85.

23. Young JB, Abraham WT, Smith AL et al. Combined cardiac resynchronization and implantable cardioversion defibrillation in advanced chronic heart failure: the MIRACLE ICD Trial. JAMA 2003; 289:2685-94

24. Lang RM, Bierig M, Devereux RB et al. Recommendations for chamber quantification. Eur J Echocardiogr 2006; 7:79-108.

25. Cazeau S, Bordachar P, Jauvert G et al. Echocardiographic modeling of cardiac dyssynchrony before and during multisite stimulation: a prospective study. Pacing Clin Electrophysiol 2003; 26:137-43. 